\title{
Quantum backaction of optical observations on Bose-Einstein condensates
}

\author{
U. Leonhardt ${ }^{1}$, T. Kiss ${ }^{2}$, and P. Piwnicki ${ }^{3}$ \\ ${ }^{1}$ Physics Department, Royal Institute of Technology (KTH), Lindstedtsvägen 24, S-10044 Stockholm, Sweden \\ ${ }^{2}$ Department of Nonlinear and Quantum Optics, Institute for Solid State Physics and Optics, P.O. Box 49, H-1525 Budapest, \\ Hungary \\ ${ }^{3}$ Abteilung für Quantenphysik, Universität Ulm, D-89069 Ulm, Germany
}

\begin{abstract}
Impressive pictures of moving Bose-Einstein condensates have been taken using phase-contrast imaging [M. R. Andrews et al., Science 273, 84 (1996)]. We calculate the quantum backaction of this measurement technique, assuming the absence of residual absorption. We find that the condensate gets gradually depleted at a universal rate that is proportional to the light intensity and to the inverse cube of the optical wave length. The fewer atoms are condensed the higher is the required intensity to see a picture, and, consequently, the higher is the induced backaction. To describe the quantum physics of phase-contrast imaging we put forward a new approach to quantum-optical propagation. We develop an effective field theory of paraxial optics in a fully quantized atomic medium.
\end{abstract}

03.75Fi, 03.65.Bz, 42.50.Dv

\section{INTRODUCTION}

Bose-Einstein condensates are macroscopic quantum states of many atoms with nearly identical wave functions. In addition to their prospects as laser-like sources of matter, the condensates will offer a new fascinating testing ground for the fundamentals of quantum mechanics, because of their macroscopic yet quantum nature. In all experiments performed so far since the pioneering breakthrough [1] Bose condensates are observed optically. One particularly intriguing method is phasecontrast imaging [2,3]. This technique allows to take impressive in situ pictures of moving droplets of Bosecondensed matter, similar to phase-contrast images of delicate living cells [4]. Figure 1 shows a simplified scheme of the method. Non-resonant laser light illuminates the sample, travels through, and attains phase shifts that are proportional to the density of the condensate. The remaining part of the apparatus serves to measure the acquired phase gradient using the non-scattered part of the incident light as a reference. Since the light is non-resonant, the condensate is hardly disturbed and, indeed, many snapshots or even entire video sequences of an individual sample have been taken without significant effect [2].

One might suspect [2], however, that the observation will nevertheless cause a quantum backaction on the condensate. Imagine that phase-contrast imaging were an ideal von-Neumann measurement of atomic positions. After each measurement the atoms were frozen in position eigenstates and their future motion were drastically altered. Why is the observed backaction much less dramatic? How does phase-contrast imaging affect the condensate? The authors of the method [2] state that "although dispersive scattering does not heat up the cloud and destroy the condensate, it will change the phase as a result of frequency shifts by the ac Stark effect." This would still allow "a nonperturbative measurement of the number of condensed atoms, which is the variable complementary to the phase (so-called quantum nondemolition measurement)."

In this paper we find that two processes contribute to the backaction. One is the phase diffusion mentioned above [2] and the second is a depletion of the condensate. The authors of Ref. 22 have already seen indications of a gradual depletion. However, they have attributed this to a residual light absorption. We find it likely that their qualitative experimental findings do in reality indicate the effect of the quantum backaction.

The physical reason for the quantum backaction is the local interaction of the meter (the incident light) with the object (the condensate). Therefore, to understand the quantum nature of phase-contrast imaging, we must study the quantum propagation of light in Bose-Einstein condensates. To a remarkable degree of accuracy [5] Bose condensates act simply as dielectric media on the incident light. In the limit of a large detuning from optical resonances of the atoms we can neglect the imaginary part of the refraction index, i.e. we consider the condensate a lossless and dispersionless dielectric medium. Quantum-optical propagation in bulk, lossless media has been studied already in detail [6]. A bulk medium, however, is hardly affected by light traveling through, and the quantum backaction was rightfully ignored so far [6]. In contrast to bulk matter, a delicate quantum gas may feel the disturbances caused by incident light. To understand actio and reactio of light in quantum gases we essay a quantum theory of paraxial light propagation in fully quantized, bosonic media. We start from the safe ground of canonical electromagnetism in dielectric media. Then we develop an approximate theory that allows an analytic calculation of the backaction. Our theory is inspired by the traditional paraxial approximation [7] to study classical light propagation. We quantize this model 
and conclude Section II with a discussion of the paraxial field commutator, a critical quantity that enters the backaction rates. Section II thus develops a general, phenomenological theory of non-resonant light propagation in matter waves.

Section III is more specific. Here we derive the master equation that describes the backaction of the propagating light on the condensate. The light plays a double role: It acts both as a meter and as a reservoir. We apply the Born-Markov approximation of reservoir theory 8 to derive a general master equation that describes the evolution of all (condensed and non-condensed) atoms. Then we restrict our attention to the condensate and analyze the two dissipative processes that turn out to appear due to the quantum backaction: phase diffusion and atomic depletion. We conclude Section III estimating the order of magnitude of the two effects. We find that the depletion far outweighs the phase diffusion. Furthermore, our estimation seems to indicate that the depletion is experimentally significant. Condensates with few atoms are especially vulnerable to optical observations, in agreement with experimental evidence [3].

\section{LIGHT AND MATTER WAVES}

\section{A. Canonical theory}

Let us start from the canonical theory of electromagnetism in a dielectric medium made of a single matter wave $\psi$. The total Lagrangian density of light and matter is (in SI units)

$$
\mathscr{L}=\frac{\epsilon_{0}}{2}\left(E^{2}-c^{2} B^{2}\right)+\frac{\epsilon_{0} \chi_{0}}{2} E^{2}|\psi|^{2}+\mathscr{L}_{M}
$$

The first term describes a free electromagnetic field characterized by the potentials $U$ and $\mathbf{A}$ that constitute the field strengths

$$
\mathbf{E}=-\frac{\partial \mathbf{A}}{\partial t}-\nabla U, \quad \mathbf{B}=\nabla \times \mathbf{A} .
$$

The second term plays a double role. The term describes both the effect of the medium $|\psi|^{2}$ on the light $E^{2}$ and the backaction of the light on the medium. The third term

$$
\mathscr{L}_{M}=\frac{i \hbar}{2}\left(\psi^{*} \dot{\psi}-\dot{\psi}^{*} \psi\right)-\frac{\hbar^{2}}{2 m}\left(\nabla \psi^{*}\right)(\nabla \psi)-V|\psi|^{2}
$$

characterizes the center-of-mass motion of the matter wave in the external potential $V$. One can show that the Euler-Lagrange equations generated by minimizing the action $\int \mathscr{L} d^{4} x$ are indeed Maxwell's equations of light in a dielectric medium with susceptibility $\epsilon_{0} \chi_{0}|\psi|^{2}$. Additionally, we obtain the Schrödinger equation of a matter wave moving in both the external potential $V$ and in the optical potential $-\epsilon_{0} \chi_{0} E^{2} / 2$. This proves that the
Lagrangian density $\mathscr{L}$ describes the physics of a single matter wave that interacts non-resonantly with a classical electromagnetic field.

Traditional quantum optics operates mostly with Hamiltonians. To find the Hamiltonian density $\mathscr{H}$ of our model we follow the canonical procedure. We calculate the functional derivatives

$$
\begin{aligned}
& \frac{\delta \mathscr{L}}{\delta \dot{\mathbf{A}}}=-\epsilon_{0}\left(1+\chi_{0}|\psi|^{2}\right) \mathbf{E} \equiv-\mathbf{D} \\
& \frac{\delta \mathscr{L}}{\delta \dot{\psi}}=\frac{i \hbar}{2} \psi^{*}, \quad \frac{\delta \mathscr{L}}{\delta \dot{\psi}^{*}}=-\frac{i \hbar}{2} \psi
\end{aligned}
$$

and get

$$
\mathscr{H} \equiv-\mathbf{D} \dot{\mathbf{A}}+\frac{i \hbar}{2}\left(\psi^{*} \dot{\psi}-\dot{\psi}^{*} \psi\right)-\mathscr{L}=\mathscr{H}_{F}+\mathscr{H}_{M}
$$

with the atomic Hamiltonian density

$$
\mathscr{H}_{M}=\frac{\hbar^{2}}{2 m}\left(\nabla \psi^{*}\right)(\nabla \psi)+V|\psi|^{2}
$$

and the electromagnetic part

$$
\mathscr{H}_{F}=\frac{\mathbf{D E}}{2}+\mathbf{D} \nabla U+\frac{\epsilon_{0} c^{2}}{2} \mathbf{B}^{2} .
$$

Observing the Maxwell equation $\nabla \mathbf{D}=0$ we ignore $\mathbf{D} \nabla U=\nabla(\mathbf{D} U)$ in $\mathscr{H}_{F}$ and arrive at the electromagnetic Hamiltonian density

$$
\mathscr{H}_{F}=\frac{\mathbf{D}^{2}}{2 \epsilon_{0}\left(1+\chi_{0}|\psi|^{2}\right)}+\frac{\epsilon_{0} c^{2}}{2} \mathbf{B}^{2} .
$$

This expression is rather unpleasant, since the interaction between light and matter appears in the denominator $\left(1+\chi_{0}|\psi|^{2}\right)$. Of course, we could represent $\left(1+\chi_{0}|\psi|^{2}\right)^{-1}$ as the geometric series $\sum_{\nu=0}^{\infty}\left(-\chi_{0}|\psi|^{2}\right)^{\nu}$, provided that the series converges. The lowest-order theory

$$
\mathscr{H}_{R}=\frac{\mathbf{D}^{2}}{2 \epsilon_{0}}+\frac{\epsilon_{0} c^{2}}{2} \mathbf{B}^{2}-\frac{\chi_{0}}{2 \epsilon_{0}} \mathbf{D}^{2}|\psi|^{2}
$$

has a familiar appearance in quantum optics, because the Hamiltonian density $\mathscr{H}_{R}$ contains the biquadratic interaction term $\mathbf{D}^{2}|\psi|^{2}$. However, if we were to use the lowest-order $\mathscr{H}_{R}$ in Section III to calculate the quantum backaction in phase-contrast imaging, we were to face an infinite result that clearly contradicts experimental evidence [2]. Can we formulate light propagation in matter waves in a different way?

\section{B. Optical Schrödinger equation}

Our canonical field theory of light and matter contains Maxwell's equations in the form of the Lagrangian $\mathscr{L}$ or, equivalently, in the Hamiltonian $\mathscr{H}$. In classical optics, however, one hardly uses Maxwell's equation to describe paraxial light propagation, but applies a characteristic approximation which is an optical analogue of the 
Schrödinger equation. Let us sketch a brief derivation of the optical Schrödinger equation []]. We start from the wave equation

$$
\begin{aligned}
& \nabla^{2} \mathbf{E}-\frac{1+\chi_{0}|\psi|^{2}}{c^{2}} \frac{\partial^{2} \mathbf{E}}{\partial t^{2}}=\nabla(\nabla \mathbf{E}), \\
& \nabla \mathbf{E}=-\nabla\left[\ln \left(1+\chi_{0}|\psi|^{2}\right)\right] \mathbf{E}
\end{aligned}
$$

(a familiar consequence of Maxwell's equations). We assume the medium $\chi_{0}|\psi|^{2}$ to be weak and to be gradually varying in space. (A mirror, for example, is excluded from this model.) We neglect the polarization mixing $\nabla(\nabla \mathbf{E})$ and consider paraxial propagation in the $z$ direction with carrier frequency $\omega_{0}$. In the following we will often refer to the wave number $k_{0}=\omega_{0} / c$ and to the wave length $\lambda=2 \pi / k_{0}$. We use the ansatz

$$
\mathbf{E}^{(+)}=\mathcal{E} \exp \left(i k_{0} z-i \omega_{0} t\right)
$$

for the positive frequency component $\mathbf{E}^{(+)}$of the electric field, and differentiate

$$
\begin{aligned}
& \frac{\partial^{2} \mathbf{E}^{(+)}}{\partial t^{2}} \approx \exp \left(i k_{0} z-i \omega_{0} t\right)\left(-2 i \omega_{0} \frac{\partial}{\partial t}-\omega_{0}^{2}\right) \mathcal{E} \\
& \frac{\partial^{2} \mathbf{E}^{(+)}}{\partial z^{2}} \approx \exp \left(i k_{0} z-i \omega_{0} t\right)\left(+2 i k_{0} \frac{\partial}{\partial z}-k_{0}^{2}\right) \mathcal{E}
\end{aligned}
$$

neglecting the slow variation of the envelope $\mathcal{E}$. Furthermore, we regard

$$
\chi_{0}|\psi|^{2} \frac{\partial^{2} \mathbf{E}}{\partial t^{2}} \approx-\omega_{0}^{2} \chi_{0}|\psi|^{2} \mathbf{E}
$$

and obtain

$$
\left[\nabla_{\perp}^{2}+2 i k_{0}\left(\frac{\partial}{\partial z}+\frac{1}{c} \frac{\partial}{\partial t}\right)+k_{0}^{2} \chi_{0}|\psi|^{2}\right] \mathcal{E}=0
$$

with $\nabla_{\perp}^{2}$ being the transversal Laplacian $\partial^{2} / \partial x^{2}+$ $\partial^{2} / \partial y^{2}$. Finally, we utilize that $(\partial / \partial z+\partial / \partial c t) \exp \left(i k_{0} z-\right.$ $\left.i \omega_{0} t\right)$ vanishes and arrive at the optical Schrödinger equation

$$
i\left(\frac{\partial}{\partial z}+\frac{1}{c} \frac{\partial}{\partial t}\right) \mathbf{E}^{(+)}=-\frac{\nabla_{\perp}^{2}}{2 k_{0}} \mathbf{E}^{(+)}-\frac{k_{0} \chi_{0}|\psi|^{2}}{2} \mathbf{E}^{(+)} .
$$

More details about the optical Schrödinger equation (and beyond) are elaborated in the comprehensive paper [7] by Marte and Stenholm.

\section{Canonical theory of paraxial propagation}

Let us describe the paraxial propagation of light in a canonical field theory. We consider a fixed polarization, i.e. a scalar electrical field strength $E^{(+)}$, and express $E^{(+)}$as

$$
E^{(+)}(\mathbf{x}, t)=\left(\frac{\hbar \omega_{0}}{2 \epsilon_{0}}\right)^{1 / 2} i \varphi(\mathbf{x}, t)
$$

The complex function $\varphi$ represents the optical field in units of the vacuum noise $\left(\hbar \omega_{0} / 2 \epsilon_{0}\right)^{1 / 2}$. One can easily verify that the Lagrangian density

$$
\begin{aligned}
\mathscr{L}_{P}= & \mathscr{L}_{F}+\mathscr{L}_{M} \\
\mathscr{L}_{F}= & \frac{i \hbar c}{2} \varphi^{*}\left(\frac{\partial}{\partial z}+\frac{1}{c} \frac{\partial}{\partial t}\right) \varphi-\frac{i \hbar c}{2} \varphi\left(\frac{\partial}{\partial z}+\frac{1}{c} \frac{\partial}{\partial t}\right) \varphi^{*} \\
& -\frac{\hbar c}{2 k_{0}}\left(\nabla_{\perp} \varphi^{*}\right)\left(\nabla_{\perp} \varphi\right)+\frac{\hbar \omega_{0}}{2} \chi_{0}|\varphi|^{2}|\psi|^{2}
\end{aligned}
$$

generates the optical Schrödinger equation (15) as an Euler-Lagrange equation for $\varphi$. This proves that $\mathscr{L}_{F}$ is a valid Lagrangian to describe paraxial optical propagation. Lagrangians are defined up to a positive prefactor (because we are only interested in their minimum). We have chosen the prefactor of $\mathscr{L}_{F}$ such that the coupling term $\hbar \omega_{0} \chi_{0}|\psi|^{2}|\varphi|^{2} / 2=\epsilon_{0} \chi_{0}|E|^{2}|\psi|^{2} \approx$ $\epsilon_{0} \chi_{0}\left(E^{(+)}+E^{(-)}\right)^{2}|\psi|^{2} / 2$ is identical to the interaction term $\epsilon_{0} \chi_{0} E^{2}|\psi|^{2} / 2$ in the canonical Lagrangian density (11). This is necessary, because the term plays the double role of describing actio and reactio of light and matter.

In order to find the Hamiltonian density we follow the royal road of canonical field theory. We calculate the functional derivatives

$$
\frac{\delta \mathscr{L}}{\delta \dot{\varphi}}=\frac{i \hbar}{2} \varphi^{*}, \quad \frac{\delta \mathscr{L}}{\delta \dot{\varphi}^{*}}=-\frac{i \hbar}{2} \varphi
$$

and obtain

$$
\begin{aligned}
\mathscr{H}_{P}= & \frac{i \hbar}{2}\left(\varphi^{*} \dot{\varphi}-\dot{\varphi}^{*} \varphi+\psi^{*} \dot{\psi}-\dot{\psi}^{*} \psi\right)-\mathscr{L}_{P} \\
= & \mathscr{H}_{M}+\mathscr{H}_{F} \\
\mathscr{H}_{F}= & \frac{i \hbar c}{2}\left(\frac{\partial \varphi^{*}}{\partial z} \varphi-\varphi^{*} \frac{\partial \varphi}{\partial z}\right)+\frac{\hbar c}{2 k_{0}}\left(\nabla_{\perp} \varphi^{*}\right)\left(\nabla_{\perp} \varphi\right) \\
& -\frac{\hbar \omega_{0}}{2} \chi_{0}|\varphi|^{2}|\psi|^{2} .
\end{aligned}
$$

The last term, $-\hbar \omega_{0} \chi_{0}|\varphi|^{2}|\psi|^{2} / 2$, has the familiar appearance of a quantum-optical light-matter interaction. The other terms of $\mathscr{H}_{F}$ describe the free paraxial propagation of the optical field.

\section{Quantum statistics}

So far we have formulated the canonical theory of a single matter wave that interacts non-resonantly with an optical field. Let us turn to the description of a Bose gas of many atoms interacting with photons. The manybody problem is conveniently formulated in the language of Second Quantization where operators $\hat{\psi}$ and $\hat{\varphi}$ describe the matter and light field, respectively. The atoms are bosons 


$$
\left[\hat{\psi}\left(\mathbf{x}_{1}\right), \hat{\psi}^{\dagger}\left(\mathbf{x}_{2}\right)\right]=\delta^{(3)}\left(\mathbf{x}_{1}-\mathbf{x}_{2}\right) .
$$

To find the Hamiltonian $\hat{H}$ of our model we integrate the Hamiltonian density $\mathscr{H}_{P}$ over three-dimensional space and apply partial integration. We obtain

$$
\begin{aligned}
\hat{H} & =\hat{H}_{R}+\hat{H}_{I}+\hat{H}_{M} \\
\hat{H}_{R} & =\hbar c \int \hat{\varphi}^{\dagger}\left(-i \frac{\partial}{\partial z}-\frac{\nabla_{\perp}^{2}}{2 k_{0}}\right) \hat{\varphi} d^{3} x \\
\hat{H}_{I} & =-\frac{\hbar \omega_{0}}{2} \chi_{0} \int \hat{\psi}^{\dagger} \hat{\psi} \hat{\varphi}^{\dagger} \hat{\varphi} d^{3} x .
\end{aligned}
$$

The Hamiltonian $\hat{H}_{R}$ describes free paraxial light propagation, $\hat{H}_{I}$ accounts for the interaction of light and matter and $\hat{H}_{M}$ describes the quantum gas of the atoms. We include atomic collisions in our model by adding an atom-atom interaction term to the potential $V$, and obtain for example [9]

$$
\hat{H}_{M}=\int\left(-\frac{\hbar^{2}}{2 m} \hat{\psi}^{\dagger} \nabla^{2} \hat{\psi}+V \hat{\psi}^{\dagger} \hat{\psi}+\frac{g}{2} \hat{\psi}^{\dagger 2} \hat{\psi}^{2}\right) d^{3} x .
$$

Note that our results turn out to be independent on the explicit form of $\hat{H}_{M}$, as long as $\hat{H}_{M}$ is able to maintain a Bose-Einstein condensate.

An important issue in our theory is the optical field commutator

$$
C\left(\mathbf{x}_{1}-\mathbf{x}_{2}\right) \equiv\left[\hat{\varphi}\left(\mathbf{x}_{1}\right), \hat{\varphi}^{\dagger}\left(\mathbf{x}_{2}\right)\right] .
$$

If $C(\mathbf{x})$ is a three-dimensional delta function we obtain the optical Schrödinger equation (15) for $\hat{\varphi}$ from the Heisenberg equation

$$
\frac{\partial \hat{\varphi}}{\partial t}=-\frac{i}{\hbar}[\hat{\varphi}, \hat{H}] .
$$

Is the commutator a delta function? The field operator $\hat{\varphi}$ is one polarization component of the photon absorption operator $\hat{\mathbf{V}}(\mathbf{x}, t)$ of Mandel and Wolf 110. The commutator $C(\mathbf{x})$ is then given in terms of the density $d \mu(\mathbf{k})$ of the employed optical modes as [11]

$$
C(\mathbf{x})=\frac{1}{(2 \pi)^{3}} \int e^{i \mathbf{k x}} d \mu(\mathbf{k}) .
$$

The crucial point is that the mode density $d \mu(\mathbf{k})$ is restricted, since we consider only those modes that obey paraxial propagation. Therefore, we cannot regard $C(\mathbf{x})$ as a three-dimensional delta function, in general. However, the commutator behaves like $\delta^{(3)}\left(\mathbf{x}_{1}-\mathbf{x}_{2}\right)$ on paraxial test functions that are supported on the spectrum of $C$ (that share the same region in $\mathbf{k}$ space). To see this we introduce the Fourier transformations $\tilde{C}(\mathbf{k})$ and $\tilde{f}(\mathbf{k})$ of the commutator and a test function $f(\mathbf{x})$, and calculate

$$
\int C\left(\mathbf{x}_{1}-\mathbf{x}_{2}\right) f\left(\mathbf{x}_{2}\right) d^{3} x_{2}
$$

$$
\begin{aligned}
& =\frac{1}{(2 \pi)^{3}} \int \tilde{C}(\mathbf{k}) \tilde{f}(\mathbf{k}) e^{i \mathbf{k} \mathbf{x}_{1}} d^{3} k \\
& =\frac{1}{(2 \pi)^{3}} \int \tilde{f}(\mathbf{k}) e^{i \mathbf{k} \mathbf{x}_{1}} d^{3} k \\
& =f\left(\mathbf{x}_{1}\right) .
\end{aligned}
$$

This is the defining property of a three-dimensional delta function. In particular, the field operator $\hat{\varphi}$ itself is a paraxial test function, and thus we can safely regard $C(\mathbf{x})$ as $\delta^{(3)}\left(\mathbf{x}_{1}-\mathbf{x}_{2}\right)$ in integrals containing $\hat{\varphi}$. In this way we obtain indeed from the Heisenberg equation (26) the optical Schrödinger equation for $\hat{\varphi}$, as is easily verified. This shows that our quantum theory of paraxial light propagation is consistent with the classical theory.

On the other hand, for test functions with a broad spatial spectrum we put

$$
\begin{aligned}
d \mu(\mathbf{k}) & =\delta\left(\omega(\mathbf{k}) / \omega_{0}-1\right) d^{3} k \\
& =\delta\left(k_{z} / k_{0}+\frac{1}{2}\left(k_{x}^{2}+k_{y}^{2}\right) / k_{0}^{2}-1\right) d^{3} k .
\end{aligned}
$$

We obtain, for example, the commutator per se

$$
\begin{aligned}
C(\mathbf{x}) & =\frac{1}{(2 \pi)^{3}} \int e^{i \mathbf{k x}} \cdot 1 \cdot d \mu(\mathbf{k}) \\
& =\frac{k_{0}^{2}}{i(2 \pi)^{2}(z-i 0)} \exp \left[\frac{i k_{0}}{2 z}\left(x^{2}+y^{2}\right)+i k_{0} z\right],
\end{aligned}
$$

see Appendix A. The commutator resembles the propagator of a free-particle wave, because $C$ satisfies

$$
\begin{aligned}
\left(-i \frac{\partial}{\partial z}-\frac{\nabla_{\perp}^{2}}{2 k_{0}}\right) C & =k_{0} C \\
C(x, y, z \rightarrow 0) & =\lambda^{-1} \delta(x) \delta(y) .
\end{aligned}
$$

Since commutators are Green's functions, we would expect this property from a paraxial quantum optics.

We have thus seen that the commutator acts differently on paraxial and on broad-spectrum test functions. The two faces of the commutator will play two different roles in the quantum backaction of phase-contrast imaging.

\section{QUANTUM BACKACTION}

\section{A. Phase-contrast imaging}

Phase-contrast imaging is a method to see transparent objects (living cells, for example) that are otherwise invisible. A transparent body does not absorb incident light but imprints a phase shift that is proportional to the local density of the object. Manipulations in the focal plane of the observing lens system transform the image of a phase object into an equivalent image of an ampli-

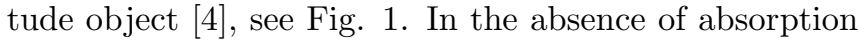
the light will not significantly affect a macroscopic body, but it might affect a delicate quantum gas. 
Formulated in the language of quantum measurement theory 12], the light is a meter of the atomic object: The incident light interacts with the atoms, becomes entangled with the atomic sample, and is finally measured in a classical apparatus (lens system and detectors). The light field is also a reservoir: The incident beam, say a plane wave, is scattered on the object into many emerging modes that form an image. The degree of collecting the scattered modes and extracting the imprinted phase information determines the performance of the measurement. The overall performance is ultimately limited by the quantum-optical phase fluctuations of the incident light and by the performance of the apparatus.

On the other hand, the average quantum backaction (averaged over all experimental runs) is solely determined by the entanglement between object and meter. This means that the average backaction is entirely independent on the performance of the measurement. We can average over the meter to calculate the backaction on the object. This averaging leads to a dissipative dynamics of the object, formulated as a quantum master equation [13.

\section{B. Master equation}

We describe the state of all atoms by the density matrix $\hat{\rho}_{I}$ in the interaction picture

$$
\hat{\rho}_{I}(t)=e^{i \hat{H}_{M} t / \hbar} \hat{\rho}(t) e^{-i \hat{H}_{M} t / \hbar}
$$

and employ standard reservoir theory 8 to derive an equation of motion for $\hat{\rho}_{I}$. The interaction between light and matter is weak and, consequently, we can apply second-order perturbation theory (the Born approximation [8]). The incident light travels through the object in a time $\tau_{c}$ that is simply given by the extension of the condensate divided by the speed of light. After $\tau_{c}$ we do not expect the light to interact further with the atomic sample. Therefore, the effective memory of the light-matter interaction is roughly given by $\tau_{c}$ and is very short. So, in addition to the Born approximation, we can apply the short-memory (Markov) approximation using an integration time $\tau_{0}$ that is much larger than the time of flight $\tau_{c}$ but is still smaller than a characteristic time of the atomic sample. We will see later in Sections $\mathrm{C}$ and D that our results do not depend on the precise value of the memory time $\tau_{0}$, provided that $\tau_{0}$ exceeds $\tau_{c}$. This justifies a posteriori our Markovian theory. We obtain in the Born-Markov approximation [8]

$$
\begin{aligned}
& \frac{d \hat{\rho}_{I}}{d t}=\frac{i}{\hbar} \operatorname{tr}_{R}\left[\hat{\rho}_{I}(t) \hat{\rho}_{I R}(t), \hat{H}_{I I}(t)\right]-\mathcal{L} \hat{\rho}_{I} \\
& \mathcal{L} \hat{\rho}_{I}=\frac{1}{\hbar^{2}} \int_{t-\tau_{0}}^{t} \operatorname{tr}_{R}\left[\hat{H}_{I I}(t),\left[\hat{H}_{I I}\left(t^{\prime}\right), \hat{\rho}_{I}(t) \hat{\rho}_{I R}(t)\right]\right] d t^{\prime}
\end{aligned}
$$

with

$$
\begin{aligned}
\hat{H}_{I I} & \equiv-\frac{\hbar \omega_{0}}{2} \chi_{0} \int \hat{\psi}_{I}^{\dagger} \hat{\psi}_{I} \hat{\varphi}_{I}^{\dagger} \hat{\varphi}_{I} d^{3} x \\
\hat{\psi}_{I}(\mathbf{x}, t) & \equiv e^{-i \hat{H}_{M} t / \hbar} \hat{\psi}(\mathbf{x}) e^{i \hat{H}_{M} t / \hbar} \\
\hat{\varphi}_{I}(\mathbf{x}, t) & \equiv e^{-i \hat{H}_{R} t / \hbar} \hat{\varphi}(\mathbf{x}) e^{i \hat{H}_{R} t / \hbar}
\end{aligned}
$$

where $\hat{\psi}$ and $\hat{\varphi}$ are Schrödinger-picture operators, and $\hat{\rho}_{I R}$ denotes the density matrix of the light field in the interaction picture

$$
\hat{\rho}_{I R}(t)=e^{i \hat{H}_{R} t / \hbar} \hat{\rho}_{R}(t) e^{-i \hat{H}_{R} t / \hbar} .
$$

The incident light is a plane wave

$$
\begin{gathered}
\operatorname{tr}_{R}\left\{\hat{\varphi}^{\dagger}\left(\mathbf{x}_{1}\right) \hat{\varphi}\left(\mathbf{x}_{2}\right) \hat{\rho}_{R}\right\}=\frac{I}{\hbar \omega_{0} c} e^{i k_{0}\left(z_{2}-z_{1}\right)} \\
\operatorname{tr}_{R}\left\{\hat{\varphi}^{\dagger}\left(\mathbf{x}_{1}\right) \hat{\varphi}^{\dagger}\left(\mathbf{x}_{2}\right) \hat{\varphi}\left(\mathbf{x}_{1}\right) \hat{\varphi}\left(\mathbf{x}_{2}\right) \hat{\rho}_{R}\right\}=\mathrm{const}
\end{gathered}
$$

that travels in the $z$ direction and carries an intensity (energy flux) of $I$. Apart from the value of the intensity we do not need to specify the quantum state of the illuminating plane wave. The atomic sample is a thin phase object, thin enough to neglect the optical diffraction inside (the $\nabla_{\perp}^{2} / 2 k_{0}$ term in Eq. (22) of the Hamiltonian $\left.\hat{H}_{R}\right)$. This means that $\hat{\varphi}_{I}$ obeys the equation

$$
i\left(\frac{\partial}{\partial z}+\frac{1}{c} \frac{\partial}{\partial t}\right) \hat{\varphi}_{I}=0
$$

with the obvious solution

$$
\hat{\varphi}_{I}\left(\mathbf{x}, t+t_{0}\right)=\hat{\varphi}_{I}\left(\mathbf{x}-c t \mathbf{e}_{z}, t_{0}\right) .
$$

The total number of atoms is fixed, i.e.

$$
\left[\hat{\rho}, \int \hat{\psi}^{\dagger} \hat{\psi} d^{3} x\right]=0 .
$$

We assume the existence of a Bose-Einstein condensate with wave function $\psi_{0}(\mathbf{x}, t)$. To describe this phenomenon, we expand the atomic annihilation operator $\hat{\psi}_{I}(\mathbf{x}, t)$ in the interaction picture into a complete, orthonormal set of atomic modes

$$
\begin{aligned}
\hat{\psi}_{I}(\mathbf{x}, t) & =\sum_{\nu} \psi_{\nu}(\mathbf{x}, t) \hat{a}_{\nu} \\
\int \psi_{\mu}^{*}(\mathbf{x}, t) \psi_{\nu}(\mathbf{x}, t) d^{3} x & =\delta_{\mu \nu} \\
\sum_{\nu} \psi_{\nu}^{*}\left(\mathbf{x}_{1}, t\right) \psi_{\nu}\left(\mathbf{x}_{2}, t\right) & =\delta^{(3)}\left(\mathbf{x}_{1}-\mathbf{x}_{2}\right) .
\end{aligned}
$$

We assume that only the $\psi_{0}$ mode (the condensate) is significantly populated, $\hat{a}_{\nu} \hat{\rho}_{I}=0$ for $\nu \neq 0$. This means that we consider the case of zero temperature and that we neglect the fluctuations [14] of the above-condensate part, for simplicity. For repulsive atom-atom interactions the spatial wave function $\psi_{0}$ of the condensate is dominated by the balance between the interatomic repulsion and the expernal potential [9]. The state of the 
condensate is described by the reduced density matrix $\hat{\rho}_{0} \equiv \operatorname{tr}_{A C} \hat{\rho}_{I}$.

For finding the master equation of the condensate state $\hat{\rho}_{0}$, we average the master equation (33) with respect to the above-condensate part. This procedure requires some lengthy yet straightforward calculations that we prefer to present in Appendix B. We obtain the result

$$
\begin{aligned}
\frac{d \hat{\rho}_{0}}{d t}= & -\operatorname{tr}_{A C} \mathcal{L} \hat{\rho}_{I}=-\mathcal{L}_{1} \hat{\rho}_{0}-\mathcal{L}_{2} \hat{\rho}_{0}, \\
\mathcal{L}_{1} \hat{\rho}_{0}= & \Gamma_{P}\left(\hat{a}_{0}^{\dagger} \hat{a}_{0} \hat{a}_{0}^{\dagger} \hat{a}_{0} \hat{\rho}_{0}-\hat{a}_{0}^{\dagger} \hat{a}_{0} \hat{\rho}_{0} \hat{a}_{0}^{\dagger} \hat{a}_{0}\right)+\text { H.c. }, \\
\mathcal{L}_{2} \hat{\rho}_{0}= & \left(\Gamma_{L}-\Gamma_{P}\right)\left(a_{0}^{\dagger} \hat{a}_{0} \hat{\rho}_{0}-\hat{a}_{0} \hat{\rho}_{0} \hat{a}_{0}^{\dagger}\right)+\text { H.c. }, \\
\Gamma_{P}= & \int_{t-\tau_{0}}^{t} \iint\left[\mathbf{x}-\mathbf{x}^{\prime}-c\left(t-t^{\prime}\right) \mathbf{e}_{z}\right] \\
& \times\left|\psi_{0}(\mathbf{x}, t)\right|^{2}\left|\psi_{0}\left(\mathbf{x}^{\prime}, t^{\prime}\right)\right|^{2} d^{3} x d^{3} x^{\prime} d t^{\prime}, \\
\Gamma_{L}= & \int_{0}^{\tau_{0}} G\left(c \tau \mathbf{e}_{z}\right) d \tau, \\
G(\mathbf{x}) \equiv & \frac{\omega_{0}}{4} \frac{\chi_{0}^{2}}{\hbar c} I C(\mathbf{x}) e^{-i k_{0} z} .
\end{aligned}
$$

We see that no macroscopic light force acts on the condensate. This is easy to understand, because light forces are caused by intensity gradients, and in our case the incident light is a plane wave with uniform intensity $I$.

We notice that two dissipative processes, $\mathcal{L}_{1} \hat{\rho}_{0}$ and $\mathcal{L}_{2} \hat{\rho}_{0}$, contribute to the backaction. The first one is a phase diffusion (see Section $\mathrm{C}$ ) and the second one is a bosonic depletion (see Section D). What is the physical origin of the two processes?

For forming an image of the condensate, the incident light is scattered on the atoms. Therefore the light transfers momentum (and energy) to the atoms. After the scattering a condensate atom may find itself in the condensate again or it may be expelled, with the (complex) rates $\Gamma_{P}$ and $\Gamma_{L}-\Gamma_{P}$, respectively. The atom that rejoined the condensate will suffer from a loss in phase coherence, causing phase diffusion, and the expelled atoms will gradually deplete the condensate. Let us look into the two processes in more detail.

\section{Phase diffusion}

Let us study the phase-diffusion $\mathcal{L}_{1} \hat{\rho}_{0}$ in the atomic number-state basis of the condensate

$$
\begin{aligned}
\left\langle m\left|\mathcal{L}_{1} \hat{\rho}_{0}\right| n\right\rangle= & {\left[i \operatorname{Im} \Gamma_{P}\left(m^{2}-n^{2}\right)+\operatorname{Re} \Gamma_{P}(m-n)^{2}\right] } \\
& \times\left\langle m\left|\hat{\rho}_{0}\right| n\right\rangle .
\end{aligned}
$$

We see that the imaginary part of $\Gamma_{P}$ governs a Hamiltonian process, i.e. a commutator of $\hat{\rho}_{0}$ with a Hermitian effective Hamiltonian that is proportional to the atom number. We may call it a Kerr-type process.
Let us turn to the complementary picture where we describe $\mathcal{L}_{1} \hat{\rho}_{0}$ in the basis of phase states [15] $|\phi\rangle \equiv$ $(2 \pi)^{-1} \sum_{n=0}^{\infty} \exp (i n \phi)|n\rangle$. We obtain

$$
\begin{aligned}
\left\langle\phi_{1}\left|\mathcal{L}_{1} \hat{\rho}_{0}\right| \phi_{2}\right\rangle= & {\left[-\operatorname{Re} \Gamma_{P}\left(\frac{\partial}{\partial \phi_{1}}+\frac{\partial}{\partial \phi_{2}}\right)^{2}\right.} \\
& \left.-i \operatorname{Im} \Gamma_{P}\left(\frac{\partial^{2}}{\partial \phi_{1}^{2}}-\frac{\partial^{2}}{\partial \phi_{2}^{2}}\right)\right]\left\langle\phi_{1}\left|\hat{\rho}_{0}\right| \phi_{2}\right\rangle .
\end{aligned}
$$

The first term is clearly a diffusion process of the atomic phase (and hence justifies our terminology of calling $\mathcal{L}_{1} \hat{\rho}_{0}$ a phase diffusion). The diffusion rate $\gamma_{P}$ is the real part of $\Gamma_{P}$.

To calculate $\gamma_{P}$ we utilize that the characteristic time of the condensate is much larger than the time of flight $\tau_{c}$ of the incident light and the integration time $\tau_{0}$. This means that we can regard $\left|\psi_{0}\left(\mathbf{x}, t^{\prime}\right)\right|^{2}$ as being equal to $\left|\psi_{0}(\mathbf{x}, t)\right|^{2}$ in the expression (44) of $\Gamma_{P}$, i.e

$$
\left|\psi_{0}\left(\mathbf{x}, t^{\prime}\right)\right|^{2}=\left|\psi_{0}(\mathbf{x}, t)\right|^{2} \equiv p_{0}(\mathbf{x}, t) .
$$

We apply the Fourier transformations

$$
\begin{aligned}
\tilde{p}_{0}(\mathbf{k}) & \equiv \int p_{0}(\mathbf{x}) e^{-i \mathbf{k} \mathbf{x}} d^{3} x \\
\tilde{G}(\mathbf{k}) & \equiv \int G(\mathbf{x}) e^{-i \mathbf{k} \mathbf{x}} d^{3} x
\end{aligned}
$$

and use the convolution theorem to obtain

$$
\gamma_{P}=\frac{1}{2(2 \pi)^{3}} \int_{-\tau_{0}}^{+\tau_{0}} \int\left|\tilde{p}_{0}(\mathbf{k}, t)\right|^{2} \tilde{G}(\mathbf{k}) e^{i c \tau k_{z}} d^{3} k d \tau
$$

Because $c \tau_{0}$ is large compared with the extension of the condensate we can approximate

$$
\gamma_{P}=\frac{\omega_{0}^{-1}}{2(2 \pi)^{3}} \int\left|\tilde{p}_{0}(\mathbf{k}, t)\right|^{2} \tilde{G}(\mathbf{k}) \delta\left(k_{z} / k_{0}\right) d^{3} k .
$$

We utilize the definition (46) of the $G$ function and find that

$$
\tilde{G}=\frac{\omega_{0}}{4} \frac{\chi_{0}^{2}}{\hbar c} I \tilde{C}\left(\mathbf{k}+k_{0} \mathbf{e}_{z}\right)
$$

with $\tilde{C}(\mathbf{k})$ being the Fourier-transformed commutator (25) expressed in terms (27) of the mode density $d \mu(\mathbf{k})$. We obtain

$$
\gamma_{P}=\frac{\pi}{4} \frac{\chi_{0}^{2}}{\hbar c} I \frac{1}{(2 \pi)^{3}} \int\left|\tilde{p}_{0}\left(\mathbf{k}-k_{0} \mathbf{e}_{z}, t\right)\right|^{2} \delta\left(\frac{k_{z}}{k_{0}}-1\right) d \mu(\mathbf{k}) .
$$

Since a typical Bose-Einstein condensate extends over many optical wave lengths, the Fourier-transformed spatial distribution $\tilde{p}_{0}(\mathbf{k})$ is narrow compared with the optical mode density. Furthermore, in Eq. (54), $\tilde{p}_{0}(\mathbf{k})$ is moved by $k_{0} \mathbf{e}_{z}$ to an area in $\mathbf{k}$ space where it overlaps 
with the optical field. The spatial distribution of the condensate thus probes the paraxial optical mode density. In this case, see Section IID, the optical-field commutator acts as a three-dimensional delta function. We set $d \mu(\mathbf{k})=d^{3} k$ and obtain, finally,

$$
\gamma_{P}=\frac{\pi}{4} \frac{\chi_{0}^{2}}{\hbar c} I \frac{k_{0}}{(2 \pi)^{3}} \int\left|\tilde{p}_{0}\left(k_{x}, k_{y}, 0, t\right)\right|^{2} d k_{x} d k_{y},
$$

or

$$
\gamma_{P}=\frac{\pi}{4} \frac{\chi_{0}^{2}}{\hbar c} I \lambda^{-1} \int\left(\int_{-\infty}^{+\infty} p_{0}(\mathbf{x}, t) d z\right)^{2} d x d y
$$

In this way we have expressed the phase-diffusion rate $\gamma_{P}$ in terms of the spatial probability distribution $p_{0}(\mathbf{x}, t)$ for a single condensed atom.

\section{Depletion}

The second backaction process of phase-contrast imaging is a familiar bosonic depletion. The Liouvillian $\mathcal{L}_{2} \hat{\rho}_{0}$ contains a Hamiltonian phase shifting that is proportional to $\operatorname{Im} \Gamma_{L}-\operatorname{Im} \Gamma_{P}$ and a depletion that occurs at the rate $\gamma_{L}-\gamma_{P}$ with $\gamma_{L} \equiv \operatorname{Re} \Gamma_{L}$. We have calculated $\gamma_{P}$ in the previous section, let us turn to $\gamma_{L}$ here. First, we proceed along similar lines as in the calculation of $\gamma_{P}$ and get

$$
\gamma_{L}=\frac{\pi}{4} \frac{\chi_{0}^{2}}{\hbar c} I \frac{1}{(2 \pi)^{3}} \int \delta\left(k_{z} / k_{0}-1\right) d \mu(\mathbf{k}) .
$$

Because

$$
\left|\tilde{p}_{0}(\mathbf{k})\right|=\left|\int p_{0}(\mathbf{x}) e^{i \mathbf{k x}} d^{3} x\right| \leq \int p_{0}(\mathbf{x}) d^{3} x=1
$$

we see from Eqs. (54) and (57) that

$$
\gamma_{L} \geq \gamma_{P}
$$

The overall depletion rate $\gamma_{L}-\gamma_{P}$ is non-negative. This satisfactory result illustrates the consistency of our theory.

Note, that we cannot replace the mode density $d \mu(\mathbf{k})$ in Eq. (57) by $d^{3} k$, as we have done in the case of phase diffusion, because in Eq. (57) we integrate $d \mu(\mathbf{k})$ over a broad spectral range. Therefore, we must use the broadspectrum behavior (30) of the commutator (27) to calculate $\gamma_{L}$. We insert the definition (46) of $G$ into the expression (45) of $\Gamma_{L}$ and obtain for $\gamma_{L}=\operatorname{Re} \Gamma_{L}$ the integral

$$
\gamma_{L}=\frac{k_{0}}{8} \frac{\chi_{0}^{2}}{\hbar c} I \int_{-c \tau_{0}}^{+c \tau_{0}} C\left(z \mathbf{e}_{z}\right) e^{-i k_{0} z} d z
$$

Then we apply the explicit formula 30 of the commutator and get

$$
\begin{aligned}
\gamma_{L} & =\frac{\pi}{4 i} \frac{\chi_{0}^{2}}{\hbar c} I \lambda^{-3} \int_{-c \tau_{0}}^{+c \tau_{0}} \frac{d z}{z-i 0} \\
& =\frac{\pi^{2}}{2} \frac{\chi_{0}^{2}}{\hbar c} I \lambda^{-3} \frac{1}{2 \pi i}\left(\oint \frac{d z}{z-i 0}-\int_{(2)} \frac{d z}{z}\right)
\end{aligned}
$$

with the contours indicated in Fig. 2. We use the residue theorem for the first integral and calculate the second explicitly. We obtain, finally,

$$
\gamma_{L}=\frac{\pi^{2}}{4} \frac{\chi_{0}^{2}}{\hbar c} I \lambda^{-3}
$$

Our result is independent on the value of the integration time $\tau_{0}$ and is therefore consistent with the assumed Markovian behavior of the quantum backaction. Furthermore, Eq. (62) does not depend on the spatial shape of the condensate. The loss rate is universal.

Equation (60) shows that the depletion rate $\gamma_{L}$ depends on the optical-field commutator $C(x, y, z)$ at $x, y=0$ integrated over $z$. So, if the commutator were a three-dimensional delta function, as in usual quantum optics, our calculations would give an infinite backaction, a result in clear contradiction to experimental evidence [2]. At this point the reader will appreciate the reason why we have developed a quantum theory of paraxial propagation in Section II. This theory employs both a biquadratic interaction (23) of light and matter and a modified commutator (30) that produces a finite backaction.

On the other hand, a first-order perturbation theory (9) of canonical electromagnetism (8) is also biquadratic in the light-matter interaction. Here the canonically conjugate variables are $\mathbf{A}$ and $\mathbf{D}$ and hence we should postulate that the commutator of $\hat{\mathbf{A}}$ and $\hat{\mathbf{D}}$ is proportional to a transversal delta function. However, this approach produces an infinite backaction. Why is perturbation theory not appropriate in the canonical Hamiltonian (8)? Remember that here we approximate the light-matter interaction $\left(1+\chi_{0}|\psi|^{2}\right)^{-1}$ by the first two terms of the geometric series $\sum_{\nu=0}^{\infty}\left(-\chi_{0}|\psi|^{2}\right)^{\nu}$. If $\psi$ is an operator $\hat{\psi}$, as it should be in the quantum statistics of matter waves, the particle nature of matter will make the density operator $\hat{\psi}^{\dagger} \hat{\psi}$ potentially large, regardless how small $\chi_{0}$ is. (For example, the density of a localized particle is a three-dimensional delta function.) Therefore, we must not approximate $\left(1+\chi_{0} \hat{\psi}^{\dagger} \hat{\psi}\right)^{-1}$ by $1-\chi_{0} \hat{\psi}^{\dagger} \hat{\psi}$ and, moreover, we must not see $\left(1+\chi_{0} \hat{\psi}^{\dagger} \hat{\psi}\right)^{-1}$ as a geometric series at all.

Let us add a more intuitive picture to our mathematical arguments. Phase-contrast imaging involves a particle-particle scattering of atoms and photons that depends on the ability of the incident photons to localize. The optical-field commutator describes potential 
photon fluctuations and therefore photon localization as well [16]. Mandel and Wolf [16] discuss in great detail that photons can manifest themselves as particles only within a volume that is bounded by the optical wave length. The finite localization volume of photons thus leads to a finite quantum backaction.

\section{E. Estimation of the effect}

How large is the backaction? Let us estimate solely the order of magnitude. First, we compare the depletion rate $\gamma_{L}$ with the phase-diffusion rate $\gamma_{P}$. The latter depends on the spatial shape of the condensate. To find a rough estimate for $\gamma_{P}$ we assume that the condensate extends over $a_{x}, a_{y}$, and $a_{z}$ in $x, y$, and $z$ direction, respectively, and we model the Fourier-transformed probability distribution by the Gaussian

$$
\tilde{p}_{0}(\mathbf{k})=\exp \left(-\frac{1}{2} a_{x}^{2} k_{x}^{2}-\frac{1}{2} a_{y}^{2} k_{y}^{2}-\frac{1}{2} a_{z}^{2} k_{z}^{2}\right) .
$$

We obtain from Eq. (55) the phase-diffusion rate

$$
\gamma_{P}=\frac{\pi^{2}}{4} \frac{\chi_{0}^{2}}{\hbar c} I \frac{1}{\lambda\left(2 \pi a_{x}\right)\left(2 \pi a_{y}\right)},
$$

or, expressed in terms of the depletion rate $\gamma_{L}$ via Eq. (62),

$$
\gamma_{P}=\gamma_{L} \frac{\lambda^{2}}{\left(2 \pi a_{x}\right)\left(2 \pi a_{y}\right)} .
$$

A typical Bose-Einstein condensate extends over many optical wave lengths $\lambda$. Therefore,

$$
\gamma_{L} \gg \gamma_{P}
$$

Depletion far outweighs phase diffusion in the backaction of phase--contrast imaging.

The depletion rate (62) is proportional to the intensity of the illumination. A moderate intensity will thus lead to a low backaction. On the other hand, low intensity light exhibits large quantum-optical phase fluctuations. To give a rough estimation, we use the uncertainty product 17]

$$
\delta \phi \delta n \approx 1
$$

of the phase and photon-number fluctuations. A coherent-state illumination has Poissonian photon statistics with a variance $\delta^{2} n$ that equals the mean $\bar{n}$, i.e.

$$
\delta^{2} \phi \approx \bar{n}^{-1}
$$

The mean photon number is the product of the average photon density $I /\left(\hbar \omega_{0} c\right)$ with the optical mode volume during the observation time $\Delta t$. The mode volume is roughly given by a cylinder of radius $\lambda$ and length $c \Delta t$. Therefore, we obtain

$$
\bar{n} \approx \frac{I}{\hbar \omega_{0} c} \pi \lambda^{2} c \Delta t=\pi \frac{\lambda^{2} I \Delta t}{\hbar \omega_{0}} .
$$

One can see an image of a phase object only when the produced phase shift $\Delta \phi$ exceeds the phase noise $\delta \phi$. Let us estimate the signal $\Delta \phi$ for a condensate of $N$ atoms with single-atom probability distribution $p_{0}(\mathbf{x})$ :

$$
\begin{aligned}
\Delta \phi & =\int\left(\mathbf{k}-\mathbf{k}_{0}\right) d \mathbf{x} \\
& \approx \frac{2 \pi}{\lambda} \int\left(\sqrt{1+\chi_{0} N p_{0}(\mathbf{x})}-1\right) d z \\
& \approx \frac{\pi}{\lambda} \chi_{0} N \eta
\end{aligned}
$$

where $\eta$ denotes the effective two-dimensional density

$$
\eta=\int p_{0}(\mathbf{x}) d z
$$

For our simple model (63) the largest value of $\eta$ is

$$
\eta=\frac{1}{2 \pi a_{x} a_{y}} .
$$

Let us compare the signal-to-noise ratio $\Delta \phi / \delta \phi$ with the induced backaction. According to our theory, the incident light depletes gradually the condensate during the observation time $\Delta t$. The average number of atoms, $\operatorname{tr}\left\{\hat{a}_{0}^{\dagger} \hat{a}_{0} \hat{\rho}_{0}\right\}$, decays by the factor of $\exp \left(-2 \gamma_{L} \Delta t\right)$. We express our result (62) for the backaction rate $\gamma_{L}$ in terms of the signal-to-noise ratio $\Delta \phi / \delta \phi$ using the estimations (68), and find

$$
\kappa \equiv 2 \gamma_{L} \Delta t \approx\left(\frac{\Delta \phi}{\delta \phi}\right)^{2}\left(N \lambda^{2} \eta\right)^{-2} .
$$

The more atoms are condensed the lower is the required intensity for producing a faithful image and, consequently, the lower is the induced backaction. On the other hand, the more macroscopic the wave function is the lower is the probability density $\eta$ and the larger is the backaction rate. We also realize from Eq. (73) that the depletion factor $\exp (-\kappa)$ is independent of the susceptibility $\chi_{0}$ (and in particular of the detuning from atomic resonances), given a fixed signal-to-noise ratio $\Delta \phi / \delta \phi$. This is easy to understand, because for a large $\chi_{0}$ the signal $\Delta \phi$ is large, but so is the backaction (62).

We obtain for the critical value $\Delta \phi \approx \delta \phi$ the depletion constant $\kappa \approx\left(N \lambda^{2} \eta\right)^{-2}$. Let us assume that $a_{x} a_{y}$ is roughly $10^{4} \lambda^{2}$. In this case the depletion constant $\kappa$ is about $N^{-2} 10^{10}$. A small condensate [3] with $N \approx 10^{3}$ atoms disappears immediately! Therefore, a continuous monitoring of the condensate is impossible, in agreement with experimental observation [3]. Larger condensates are more robust. For $N \approx 10^{6}$ atoms [2] we obtain a decay factor $\exp (-\kappa)$ of about $1-\kappa \approx 1-10^{-2}$. The depletion is in the order of one percent which agrees well 
with the experimental observation [2]. However, the authors of Ref. [2] have interpreted the decay of the condensate as an residual absorption effect. Our calculations seem to indicate that the quantum backaction of phasecontrast imaging has caused the observed depletion via the momentum transfer of the illuminating light quanta.

Finally, we remark that the use of appropriately squeezed 18] illumination would enhance the signal-tonoise ratio at a given, moderate quantum backaction.

\section{SUMMARY}

Phase-contrast imaging [2] induces a quantum backaction on Bose-Einstein condensates. The backaction consists of two dissipative processes: a phase diffusion and a gradual depletion of the condensate. If phase-contrast imaging were a quantum nondemolition measurement of the condensate density, solely phase diffusion would occur. We have shown, however, that the depletion is the dominant process. Our estimations indicate that the quantum backaction is indeed an observable effect that limits the otherwise destructionless character of phasecontrast imaging [2]. Condensates with a few number of atoms are especially vulnerable to optical observations.

In our theoretical study we ignored entirely the absorption of light (the imaginary part of the refractive index) and we focused our attention on the quantum backaction. This is justified in the limit of strong detuning, because here the absorption virtually vanishes. Note that our theory is only valid as long as the condensate is still significantly populated. We have assumed a priori the existence and the dominance of the condensate.

We developed a quantum theory of paraxial light propagation in many-atom samples that are off-resonant with respect to the light. This theory goes beyond the immediate purpose of the present paper and may find wider application in the fascinating subject of combining concepts of quantum measurement theory with quantum-optical propagation and the statistical theory of quantum gases.

\section{ACKNOWLEDGMENTS}

We acknowledge the support of the research consortium Quantum Gases of the Deutsche Forschungsgemeinschaft. U. L. thanks the Alexander von Humboldt Foundation and the Göran Gustafsson Stiftelse. T. K. was supported by an Eötvös Fellowship and by the grants F017381 and T023777 of the National Research Fund of Hungary (OTKA). T. K. is grateful to W. P. Schleich for his kind hospitality in Ulm.

\section{APPENDIX A}

In this appendix we calculate explicitly the optical field commutator $C(\mathbf{x})$ and we discuss a few general properties of $C(\mathbf{x})$. We start from the expression (27) of $C(\mathbf{x})$ in terms of the mode density $d \mu(\mathbf{k})$,

$$
\begin{aligned}
C(\mathbf{x}) & =\frac{1}{(2 \pi)^{3}} \int e^{i \mathbf{k x}} d \mu(\mathbf{k}), \\
d \mu(\mathbf{k}) & =\delta\left(k_{z} / k_{0}+\frac{1}{2}\left(k_{x}^{2}+k_{y}^{2}\right) / k_{0}^{2}-1\right) d^{3} k .
\end{aligned}
$$

To calculate the Fourier integral we introduce dimensionless variables, $k_{x}=k_{0} \zeta_{x}, k_{y}=k_{0} \zeta_{y}$ with

$$
k_{z}=k_{0}-\frac{1}{2} k_{0}\left(\zeta_{x}^{2}+\zeta_{y}^{2}\right)
$$

and obtain

$$
\begin{array}{r}
C(\mathbf{x})=\frac{k_{0}^{3}}{(2 \pi)^{3}} e^{i k_{0} z} \int_{-\infty}^{+\infty} e^{-i \zeta_{x}^{2} k_{0}(z-i 0) / 2+i k_{0} x \zeta_{x}} d \zeta_{x} \\
\times \int_{-\infty}^{+\infty} e^{-i \zeta_{y}^{2} k_{0}(z-i 0) / 2+i k_{0} y \zeta_{y}} d \zeta_{y}
\end{array}
$$

In Eq. 76 we have given $z$ an infinitesimally small yet negative imaginary part $-i 0$ to ensure that the integrals converge. The calculation of Eq. (76) is straightforward, and we obtain the result

$$
C(\mathbf{x})=\frac{k_{0}^{2}}{i(2 \pi)^{2}(z-i 0)} \exp \left[\frac{i k_{0}}{2 z}\left(x^{2}+y^{2}\right)+i k_{0} z\right] \text {. }
$$

Let us discuss a few elementary properties of the paraxial commutator. First, we see from the mode density in Eq. (74) that $C(\mathbf{x})$ obeys the partial differential equation

$$
\left(-i \frac{\partial}{\partial z}-\frac{\nabla_{\perp}^{2}}{2 k_{0}}\right) C=k_{0} C
$$

The commutator behaves like a paraxial monochromatic wave that travels freely in space without being influenced by a medium. Furthermore, when $z$ approaches zero, the commutator $C(x, y, z \rightarrow 0)$ vanishes effectively for $x, y \neq 0$, because the exponential function in formula (77) is highly oscillating in this limit. To see what happens at $x, y=0$ we integrate $C(x, y, z \rightarrow 0)$ with respect to $x$ and $y$. Since

$$
\begin{aligned}
\int_{-\infty}^{+\infty} \int_{-\infty}^{+\infty} & \exp \left[\frac{i k_{0}}{2 z}\left(x^{2}+y^{2}\right)\right] d x d y \\
= & 2 \pi \int_{0}^{\infty} \exp \left(\frac{i k_{0}}{2 z} r^{2}\right) d\left(\frac{1}{2} r^{2}\right) \\
& =2 \pi i \frac{z}{k_{0}}
\end{aligned}
$$

we obtain, finally,

$$
C(x, y, z \rightarrow 0)=\frac{k_{0}}{2 \pi} \delta(x) \delta(y) .
$$

The commutator $C(\mathbf{x})$ appears as a paraxial Green's function. 


\section{APPENDIX B}

In this appendix we derive the master equation (41) that describes the quantum backaction of phase-contrast imaging on Bose-Einstein condensates. We start from the general master equation (33) and proceed in two steps. First, we reformulate the general master equation (33) utilizing the fact (36) that the incident light is a plane wave and using the conservation (39) of the total number of atoms. Then we average the resulting Liouvillian with respect to the above-condensate part. This, finally, gives us the desired master equation for the condensate.

To begin, let us first consider the Hamiltonian term $\operatorname{tr}_{R}\left[\hat{\rho}_{I} \hat{\rho}_{I R}, \hat{H}_{I I}\right]$ in Eq. (33) that describes the average light force on the atomic sample. We calculate the commutator of the interaction Hamiltonian and the total density matrix in the Schrödinger picture using Eq. (36) and the conservation of the total number of atoms, Eq. (39),

$$
\operatorname{tr}_{R}\left[\hat{H}_{I}, \hat{\rho} \hat{\rho}_{R}\right]=\frac{I}{2 c} \chi_{0}\left[\int \hat{\psi}^{\dagger} \hat{\psi} d^{3} x, \hat{\rho}\right]=0 .
$$

Consequently, we get in the interaction picture

$$
\operatorname{tr}_{R}\left[\hat{H}_{I I}, \hat{\rho}_{I} \hat{\rho}_{I R}\right]=0
$$

The overall light force of an uniform illumination vanishes.

Let us turn to the Liouvillian part $\mathcal{L} \hat{\rho}_{I}$ of the master equation (33). First we calculate the correlation functions (36) of the incident light in the interaction picture. We neglect optical diffraction inside the atomic sample, see Eq. (38), and apply Eq. (36) to get

$$
\begin{aligned}
& \operatorname{tr}_{R}\left\{\hat{\varphi}_{I}^{\dagger}\left(\mathbf{x}_{\mathbf{1}}, t_{1}\right) \hat{\varphi}_{I}\left(\mathbf{x}_{\mathbf{2}}, t_{2}\right) \hat{\rho}_{I R}\left(t_{2}\right)\right\} \\
& =\operatorname{tr}_{R}\left\{\hat{\varphi}_{I}^{\dagger}\left(\mathbf{x}_{\mathbf{1}}-c\left(t_{1}-t_{2}\right) \mathbf{e}_{z}, t_{2}\right) \hat{\varphi}_{I}\left(\mathbf{x}_{\mathbf{2}}, t_{2}\right) \hat{\rho}_{I R}\left(t_{2}\right)\right\} \\
& =\operatorname{tr}_{R}\left\{e^{-i \hat{H}_{R} t_{2}} \hat{\varphi}^{\dagger}\left(\mathbf{x}_{\mathbf{1}}-c\left(t_{1}-t_{2}\right) \mathbf{e}_{z}\right) \hat{\varphi}\left(\mathbf{x}_{\mathbf{2}}\right) \hat{\rho}_{R}\left(t_{2}\right) e^{i \hat{H}_{R} t_{2}}\right\} \\
& =\operatorname{tr}_{R}\left\{\hat{\varphi}^{\dagger}\left(\mathbf{x}_{\mathbf{1}}-c\left(t_{1}-t_{2}\right) \mathbf{e}_{z}\right) \hat{\varphi}\left(\mathbf{x}_{\mathbf{2}}\right) \hat{\rho}_{R}\left(t_{2}\right)\right\} \\
& =\frac{I}{\hbar \omega_{0} c} \exp \left[i k_{0}\left(z_{2}-z_{1}+c\left(t_{1}-t_{2}\right)\right)\right],
\end{aligned}
$$

and along similar lines

$$
\begin{aligned}
& \operatorname{tr}_{R}\left\{\hat{\varphi}_{I}^{\dagger}\left(\mathbf{x}_{\mathbf{1}}, t_{1}\right) \hat{\varphi}_{I}^{\dagger}\left(\mathbf{x}_{\mathbf{2}}, t_{2}\right) \hat{\varphi}_{I}\left(\mathbf{x}_{\mathbf{1}}, t_{1}\right) \hat{\varphi}_{I}\left(\mathbf{x}_{\mathbf{2}}, t_{2}\right) \hat{\rho}_{I R}\right\} \\
& =\text { const }
\end{aligned}
$$

To evaluate the Liouvillian $\mathcal{L} \hat{\rho}_{I}$ in Eq. (33) we calculate the double commutator using the shorthand notation $\hat{\psi}_{i} \equiv \hat{\psi}_{I}\left(\mathbf{x}_{i}, t_{i}\right), \hat{\varphi}_{i} \equiv \hat{\varphi}_{I}\left(\mathbf{x}_{i}, t_{i}\right)$,

$$
\begin{aligned}
& \operatorname{tr}_{R}\left\{\left[\hat{H}_{I I}\left(t_{1}\right),\left[\hat{H}_{I}\left(t_{2}\right), \hat{\rho}_{I} \hat{\rho}_{I R}\right]\right]\right\} \\
& =\frac{\hbar^{2} \omega_{0}^{2}}{4} \chi_{0}^{2} \iint \operatorname{tr}_{R}\left\{\hat{\psi}_{1}^{\dagger} \hat{\psi}_{1} \hat{\varphi}_{1}^{\dagger} \hat{\varphi}_{1} \hat{\psi}_{2}^{\dagger} \hat{\psi}_{2} \hat{\varphi}_{2}^{\dagger} \hat{\varphi}_{2} \hat{\rho}_{I} \hat{\rho}_{I R}\right.
\end{aligned}
$$

$$
\begin{aligned}
& -\hat{\psi}_{1}^{\dagger} \hat{\psi}_{1} \hat{\varphi}_{1}^{\dagger} \hat{\varphi}_{1} \hat{\rho}_{I} \hat{\rho}_{I R} \hat{\psi}_{2}^{\dagger} \hat{\psi}_{2} \hat{\varphi}_{2}^{\dagger} \hat{\varphi}_{2} \\
& -\hat{\psi}_{2}^{\dagger} \hat{\psi}_{2} \hat{\varphi}_{2}^{\dagger} \hat{\varphi}_{2} \hat{\rho}_{I} \hat{\rho}_{I R} \hat{\psi}_{1}^{\dagger} \hat{\psi}_{1} \hat{\varphi}_{1}^{\dagger} \hat{\varphi}_{1} \\
& \left.+\hat{\rho}_{I} \hat{\rho}_{I R} \hat{\psi}_{2}^{\dagger} \hat{\psi}_{2} \hat{\varphi}_{2}^{\dagger} \hat{\varphi}_{2} \hat{\psi}_{1}^{\dagger} \hat{\psi}_{1} \hat{\varphi}_{1}^{\dagger} \hat{\varphi}_{1}\right\} \\
& \times d^{3} x_{1} d^{3} x_{2} \\
= & \frac{\hbar^{2} \omega_{0}^{2}}{4} \chi_{0}^{2} \iint\left(\hat{\psi}_{1}^{\dagger} \hat{\psi}_{1} \hat{\psi}_{2}^{\dagger} \hat{\psi}_{2} \hat{\rho}_{I}-\hat{\psi}_{2}^{\dagger} \hat{\psi}_{2} \hat{\rho}_{I} \hat{\psi}_{1}^{\dagger} \hat{\psi}_{1}\right) \\
& \times \operatorname{tr}_{R}\left\{\hat{\varphi}_{1}^{\dagger} \hat{\varphi}_{1} \hat{\varphi}_{2}^{\dagger} \hat{\varphi}_{2} \hat{\rho}_{I R}\right\} d^{3} x_{1} d^{3} x_{2}+\text { H.c. } .
\end{aligned}
$$

Then we utilize the commutator relation of the radiation field (25) and the diffraction-less propagation (38) in the interaction zone to write $\operatorname{tr}_{R}\left\{\hat{\varphi}_{1}^{\dagger} \hat{\varphi}_{1} \hat{\varphi}_{2}^{\dagger} \hat{\varphi}_{2} \hat{\rho}_{I R}\right\}$ in normal order,

$$
\begin{aligned}
& \operatorname{tr}_{R}\left\{\hat{\varphi}_{1}^{\dagger} \hat{\varphi}_{1} \hat{\varphi}_{2}^{\dagger} \hat{\varphi}_{2} \hat{\rho}_{I R}\right\} \\
& =\operatorname{tr}_{R}\left\{\hat{\varphi}_{1}^{\dagger} \hat{\varphi}_{2}^{\dagger} \hat{\varphi}_{1} \hat{\varphi}_{2} \hat{\rho}_{I R}\right\} \\
& \quad+\operatorname{tr}_{R}\left\{\hat{\varphi}_{1}^{\dagger} \hat{\varphi}_{2} \hat{\rho}_{I R}\right\} C\left(\mathbf{x}_{\mathbf{1}}-\mathbf{x}_{\mathbf{2}}-c\left(t_{1}-t_{2}\right) \mathbf{e}_{z}\right) .
\end{aligned}
$$

The normally-ordered correlation function (84) is constant, and so $\operatorname{tr}_{R}\left\{\hat{\varphi}_{1}^{\dagger} \hat{\varphi}_{2}^{\dagger} \hat{\varphi}_{1} \hat{\varphi}_{2} \hat{\rho}_{I R}\right\}$ does not contribute to the double commutator (85), when we take into account the atom-number conservation (39). To proceed with the remaining term, we utilize Eq. (83) to express the correlation function $\operatorname{tr}_{R}\left\{\hat{\varphi}_{1}^{\dagger} \hat{\varphi}_{2} \hat{\rho}_{I R}\right\}$, define in Eq. (46) the function $G(\mathbf{x})$, and get

$$
\begin{aligned}
\mathcal{L} \hat{\rho}_{I}= & \int_{-\infty}^{t} \iint\left(\hat{\psi}_{1}^{\dagger} \hat{\psi}_{1} \hat{\psi}_{2}^{\dagger} \hat{\psi}_{2} \hat{\rho}_{I}-\hat{\psi}_{2}^{\dagger} \hat{\psi}_{2} \hat{\rho}_{I} \hat{\psi}_{1}^{\dagger} \hat{\psi}_{1}\right) \\
& \times G\left(\mathbf{x}_{\mathbf{1}}-\mathbf{x}_{\mathbf{2}}-c\left(t-t^{\prime}\right) \mathbf{e}_{z}\right) d^{3} x_{1} d^{3} x_{2} d t^{\prime}+\text { H.c. }
\end{aligned}
$$

In this way we have found the Liouvillian that describes the quantum backaction of an uniform illumination on the total atomic sample.

So far we have not made any essential assumption on the state of the atoms and in particular we have not exploited the fact that the atoms are Bose-condensed. To incorporate Bose-Einstein condensation we expand the atomic annihilation operator $\hat{\psi}_{I}$ in the interaction picture in a complete, orthonormal basis of atomic modes, see Eq. (40). We assume that only $\psi_{0}$ is significantly occupied and average with respect to the above-condensate part being in the vacuum state. (We utilize that $\hat{a}_{\nu} \hat{\rho}_{I}$ vanishes for $\nu \neq 0$.) For this we must calculate some atomic correlation functions that occur in the Liouvillian (87). We abbreviate $\operatorname{tr}_{A C} \hat{\rho}_{I}$ by $\hat{\rho}_{0}$, and find

$$
\begin{aligned}
& \operatorname{tr}_{A C}\left\{\hat{\psi}_{1}^{\dagger} \hat{\psi}_{2}^{\dagger} \hat{\psi}_{1} \hat{\psi}_{2} \hat{\rho}_{I}\right\} \\
& =\left(\hat{a}_{0}^{\dagger}\right)^{2} \hat{a}_{0}^{2}\left|\psi_{0}\left(\mathbf{x}_{1}, t_{1}\right)\right|^{2}\left|\psi_{0}\left(\mathbf{x}_{2}, t_{2}\right)\right|^{2} \hat{\rho}_{0}, \\
& \operatorname{tr}_{A C}\left\{\hat{\psi}_{1}^{\dagger} \hat{\psi}_{1} \hat{\rho}_{I}\right\}=\hat{a}_{0}^{\dagger} \hat{a}_{0}\left|\psi_{0}(\mathbf{x}, t)\right|^{2} \hat{\rho}_{0}, \\
& \operatorname{tr}_{A C}\left\{\hat{\psi}_{2}^{\dagger} \hat{\psi}_{2} \hat{\rho}_{I} \hat{\psi}_{1}^{\dagger} \hat{\psi}_{1}\right\}
\end{aligned}
$$




$$
\begin{aligned}
&= \operatorname{tr}_{A C}\left\{\hat{\psi}_{2}^{\dagger} \hat{a}_{0} \hat{\rho}_{I} \hat{a}_{0}^{\dagger} \hat{\psi}_{1}\right\} \psi_{0}\left(\mathbf{x}_{2}, t_{2}\right) \psi_{0}^{*}\left(\mathbf{x}_{1}, t_{1}\right) \\
& \operatorname{tr}_{A C}\left\{\hat{\psi}_{2}^{\dagger} \hat{a}_{0} \hat{\rho}_{I} \hat{a}_{0}^{\dagger} \hat{\psi}_{1}\right\} \\
&=\sum_{\nu \mu} \operatorname{tr}_{A C}\left\{\hat{a}_{\nu}^{\dagger} \hat{a}_{0} \hat{\rho}_{I} \hat{a}_{0}^{\dagger} \hat{a}_{\mu}\right\} \psi_{\nu}^{*}\left(\mathbf{x}_{2}, t_{2}\right) \psi_{\mu}\left(\mathbf{x}_{1}, t_{1}\right) \\
&=\sum_{\nu} \operatorname{tr}_{A C}\left\{\hat{a}_{\nu}^{\dagger} \hat{a}_{0} \hat{\rho}_{I} \hat{a}_{0}^{\dagger} \hat{a}_{\nu}\right\} \psi_{\nu}^{*}\left(\mathbf{x}_{2}, t_{2}\right) \psi_{\nu}\left(\mathbf{x}_{1}, t_{1}\right) \\
&=\hat{a}_{0}^{\dagger} \hat{a}_{0} \hat{\rho}_{0} \hat{a}_{0}^{\dagger} \hat{a}_{0} \psi_{0}^{*}\left(\mathbf{x}_{2}, t_{2}\right) \psi_{0}\left(\mathbf{x}_{1}, t_{1}\right) \\
& \quad+\hat{a}_{0} \hat{\rho}_{0} \hat{a}_{0}^{\dagger} \sum_{\nu \neq 0} \psi_{\nu}^{*}\left(\mathbf{x}_{2}, t_{2}\right) \psi_{\nu}\left(\mathbf{x}_{1}, t_{1}\right) \\
&= \hat{a}_{0}^{\dagger} \hat{a}_{0} \hat{\rho}_{0} \hat{a}_{0}^{\dagger} \hat{a}_{0} \psi_{0}^{*}\left(\mathbf{x}_{2}, t_{2}\right) \psi_{0}\left(\mathbf{x}_{1}, t_{1}\right)+\hat{a}_{0} \hat{\rho}_{0} \hat{a}_{0}^{\dagger} \delta^{(3)}\left(\mathbf{x}_{1}-\mathbf{x}_{2}\right) \\
&-\hat{a}_{0} \hat{\rho}_{0} \hat{a}_{0}^{\dagger} \psi_{0}^{*}\left(\mathbf{x}_{2}, t_{2}\right) \psi_{0}\left(\mathbf{x}_{1}, t_{1}\right) .
\end{aligned}
$$

We apply the results 88-91) to obtain after averaging of the Liouvillian (87) with respect to the above-condensate part the final master equation (41).

[1] M. H. Anderson et al., Science 269, 198 (1995); C. C. Bradley et al., Phys. Rev. Lett. 75, 1687 (1995); K. B. Davis et al., ibid. 75, 3969 (1995).

[2] M. R. Andrews et al., Science 273, 84 (1996).

[3] C. C. Bradley, C. A. Sackett, and R. G. Hulet, Phys. Rev. Lett. 78, 985 (1997); C. A. Sackett et al., Appl. Phys. B 65, 433 (1997).

[4] E. Hecht, Optics (Addison-Wesley, Reading, 1989).

[5] O. Morice, Y. Castin, and J. Dalibard, Phys. Rev. A 51, 3896 (1995). For the theory of light propagation in Bose condensates see also B. V. Svistunov and G. V. Shlyapnikov, Sov. Phys. JETP 70, 460 (1990); ibid. 71, 71, (1990); H. D. Politzer, Phys. Rev. A 43, 6444 (1991); M. Lewenstein and L. You, Phys. Rev. Lett. 71, 1339 (1993); J. Javanainen, ibid. 72, 2375 (1994); M. Lewenstein et al., Phys. Rev. A 50, 2207 (1994); L. You et al., ibid. 51, 4712 (1995); 53, 329 (1996); J. Javanainen and J. Ruostekoski, ibid. 52, 3033 (1995); R. Graham and D. F. Walls, Phys. Rev. Lett. 76, 1774 (1996); A. Csordás et al., Phys. Rev. A 54, R2543 (1996); J. Ruostekoski and J. Javanainen, ibid. 55, 513 (1997); J. Ruostekoski and D. F. Walls, ibid. 56, 2996 (1997).

[6] L. Knöll, W. Vogel, and D.-G. Welsch, Phys. Rev. A 36, 3803 (1987) R. J. Glauber and M. Lewenstein, ibid. 43, 467 (1991); L. Knöll, W. Vogel, and D.-G. Welsch, ibid. 43, 543 (1991); L. Knöll and D.-G. Welsch, Progr. Quant. Electron. 16, 135 (1992).

[7] M. A. M. Marte and S. Stenholm, Phys. Rev. A 56, 2940 (1997).

[8] See e.g. H. Carmichael, An Open Systems Approach to Quantum Optics (Springer, Berlin, 1993), Chapter 1.2.

[9] See e.g. F. Dalfovo, S. Giorgini, L. P. Pitaevskii, and S. Stringari, Rev. Mod. Phys. (in press).

[10] See Ref. [19], Chapter 12.3 and Chapter 12.11.1.
[11] Mandel and Wolf obtain a commutation relation of the vectorial photon absorption operator $\hat{\mathbf{V}}$ in Eq. (12.11-5) of Ref. 119]. Our operator $\hat{\varphi}$ is one polarization component of $\mathbf{V}$. We specialize Eq. (12.11-5) to one component and get the commutation relation

$$
C(\mathbf{x})=\frac{1}{L^{3}} \sum_{[\mathbf{k}]} e^{i \mathbf{k x}} \equiv \frac{1}{(2 \pi)^{3}} \int e^{i \mathbf{k x}} d \mu(\mathbf{k}) .
$$

for $C\left(\mathbf{x}_{1}-\mathbf{x}_{2}\right) \equiv\left[\hat{\varphi}\left(\mathbf{x}_{1}\right), \hat{\varphi}^{\dagger}\left(\mathbf{x}_{2}\right]\right.$. Here $L$ denotes the usual quantization length that tends to infinity. We have introduced the mode density $d \mu(\mathbf{k})$ to replace the sum of modes by an integral.

[12] For the theory of continuous quantum measurements see C. M. Caves and G. J. Milburn, Phys. Rev. A 36, 5543 (1987). See also A. Barchielli, Phys. Rev. A 34, 1642 (1986) and V. P. Belavkin, J. Phys. A 22, L1109 (1989) and references cited therein. For an application to BEC see J. R. Corney and G. J. Milburn, Phys. Rev. A 58, 2399 (1998).

[13] Our theoretical approach is related to the quantum kinetic theory of weakly interacting Bose gases, see C. W. Gardiner and P. Zoller, Phys. Rev. A 55, 2902 (1997); C. W. Gardiner et al., Phys. Rev. Lett. 79, 1793 (1997); D. Jaksch et al., Phys. Rev. A 56, 575 (1997); C. W. Gardiner and P. Zoller, ibid. 58, 536 (1998); D. Jaksch et al., ibid. 58, 1450 (1998); C. W. Gardiner et al., Phys. Rev. Lett. 81, 5266 (1998).

[14] Analytic results on the fluctuations of the abovecondensate part have been obtained by S. Stringari, Phys. Rev. Lett. 77, 2360 (1996); M. Fliesser et al., Phys. Rev. A 56, R2533 (1997); P. Öhberg et al., ibid. 56, R3346 (1997). For numerical studies see e.g. M. Edwards et al., Phys. Rev. Lett. 77, 1671 (1996); K. G. Singh and D. S. Rokhsar, ibid. 77, 1676 (1996); L. You, W. Houston, and M. Lewenstein, Phys. Rev. A 55, R1581 (1997).

[15] See e.g. U. Leonhardt, Measuring the Quantum State of Light, (Cambridge University Press, Cambridge, 1997), Chapter 6.3.

[16] See e.g. the discussion in Chapter 12.11. of Mandel and Wolf 119 and the references cited therein.

[17] The uncertainty relation (67) between number and phase holds only in an approximative sense for quasiclassical states of light. For a critical review of the quantumoptical phase problem see R. Lynch, Phys. Rep. 256, 367 (1995).

[18] See e.g. G. Breitenbach et al., Nature 387, 471 (1997).

[19] L. Mandel and E. Wolf, Optical coherence and quantum optics, (Cambridge University Press, Cambridge, 1995). 


\section{FIGURE CAPTION}

Figure 1: Scheme of phase-contrast imaging [4]. To produce an image $I$ of a transparent object $O$ the non-scattered light is phase--shifted (phase-contrast microscopy) or blocked (dark-ground method [2]) in the focal plane $F$ of the magnifying lens $L$.

Figure 2: Integration contours of Eq. (61) in the complex $z$ plane. The closed contour (1) \& (2) encircles a $(z-i 0)^{-1}$ singularity (dot). 


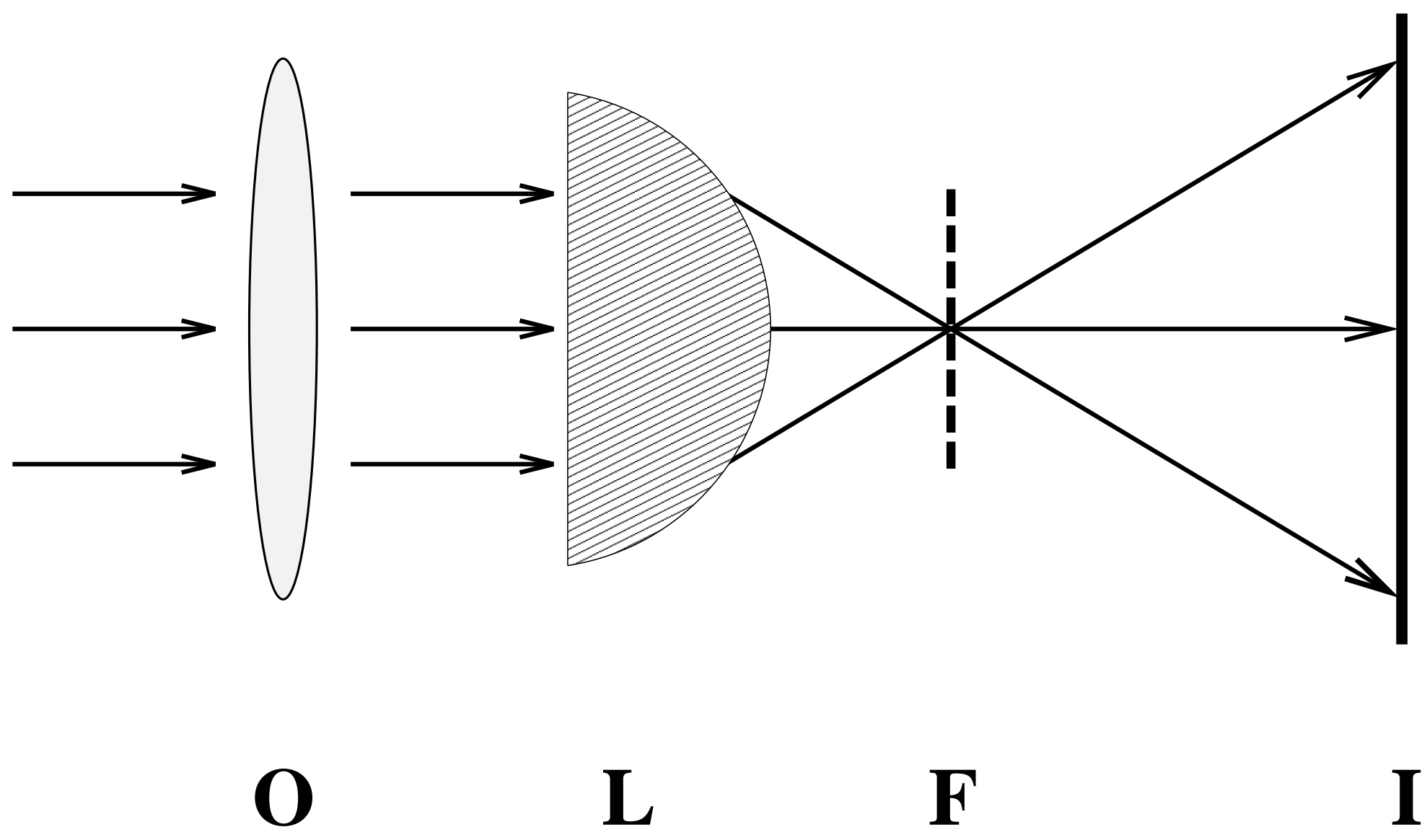




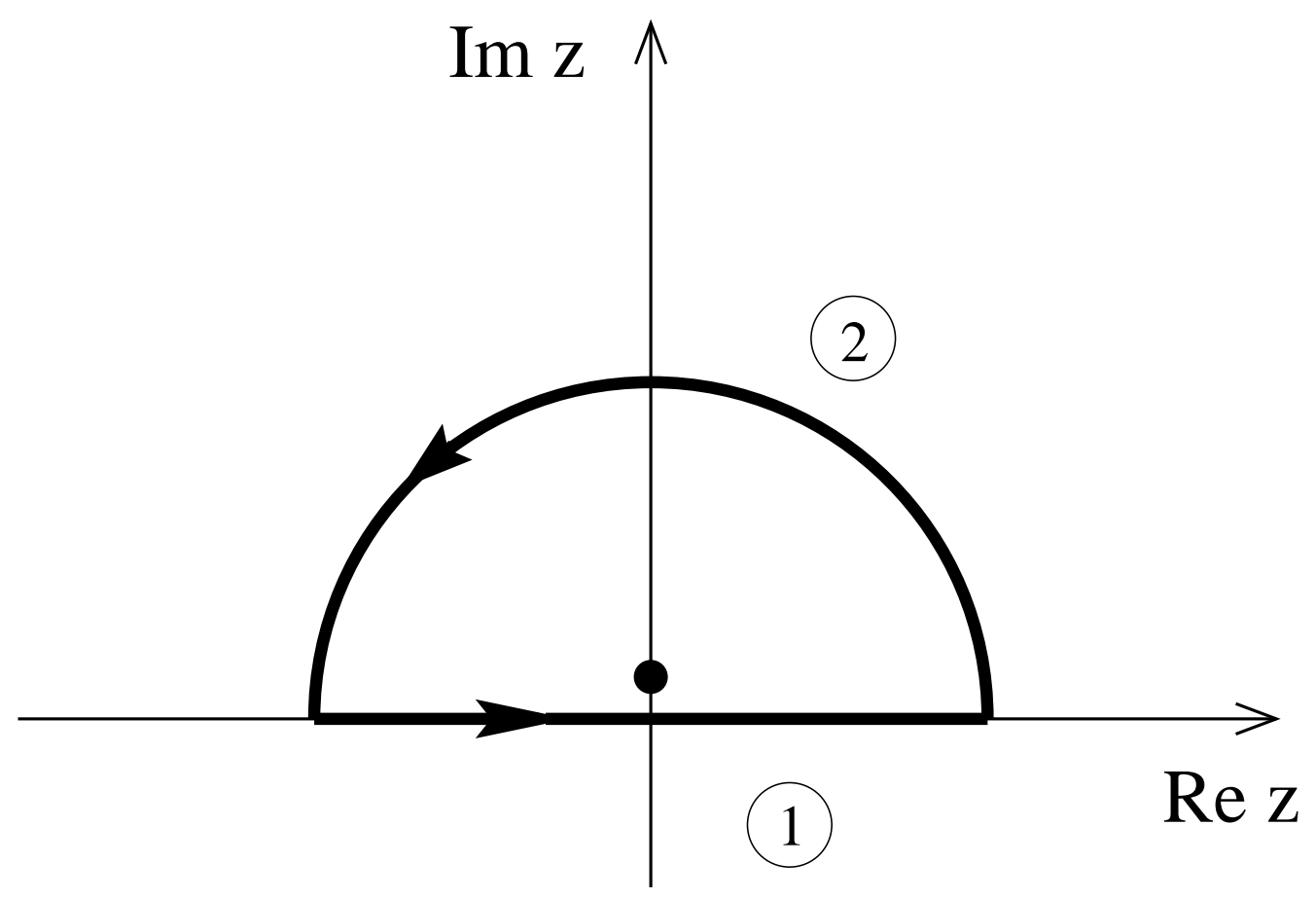

